\title{
Fault low velocity zones deduced by trapped waves and their relation to earthquake rupture processes
}

\author{
Yasuto Kuwahara and Hisao Ito \\ Geological Survey of Japan, National Institute of Advanced Industrial Science and Technology, \\ AIST Tsukuba Central 7, 1-1-1 Higashi, Tsukuba, Ibaraki 305-8567, Japan
}

(Received December 28, 2001; Revised June 19, 2002; Accepted June 26, 2002)

\begin{abstract}
Detailed structures of fault low velocity zones (LVZ) have been studied by analyzing fault-zone trapped waves at various active faults. These studies demonstrate that widths of the fault LVZ are ranging from an order of $10 \mathrm{~m}$ to a few hundred meters. In order to evaluate the effect of fault LVZ on the earthquake rupture process, a model of the LVZ related to the plastic deformation around an edge of propagating earthquake rupture is proposed. In this study, earthquake rupture processes are regarded as Mode III crack propagation. The LVZ is identical to the fault damaged zone which is related to plastic deformation in the vicinity of the crack tip. The Mode III crack analysis gives a simple relationship between the width of the low velocity zone, the breakdown stress drop at the crack tip, and characteristic slip distance $d_{0}$ in friction laws. The parameters applicable to the Nojima fault producing the 1995 Hyogo-ken Nanbu earthquake show that the breakdown stress drop is 10 times larger than the static stress drop and $d_{0}$ is about $10 \mathrm{~cm}$. These values are consistent with the value obtained by the other study for the Nojima fault so the present model is applicable for considering earthquake rupture within the damaged zone.
\end{abstract}

\section{Introduction}

Recent studies have shown that seismic waves from small earthquakes are trapped in low velocity zones (LVZ) of major crustal faults (e.g. Li et al., 1990, 1994; Ito and Kuwahara, 1996). They showed that the LVZ exists at seismogenic depths and extends to the earth surface for active faults generating large earthquakes with magnitudes larger than about 7. The existence of an LVZ in the Nojima faults associated with the 1995 Hyogo-ken Nanbu earthquake $\left(\mathrm{M}_{\mathrm{JMA}}=7.2\right)$, at a depth of about $700 \mathrm{~m}$, was demonstrated by fault drilling (Ito et al., 1996). The authors reported that the LVZ of the Nojima fault has a width of about $50 \mathrm{~m}$ and reductions in $\mathrm{P}$ - and S-wave velocities of $30 \%$ and $40 \%$ respectively on average, and that the LVZ is characterized by altered and deformed rocks. Mooney and Ginzburg (1986) also showed that LVZ's reflect mineralogic alteration and intense fracturing associated with faulting. These studies indicate the presence of a zone of altered rock generated from past earthquake ruptures. It is noted that such zones have also been characterized by electro-magnetic methods suggesting high porosity with fluid in the zone (e.g. Unsworth et al., 1999; Fujita and Ikuta, 2000). Since the LVZ is the earthquake rupture zone, it appears plausible that the presence of the LVZ has significant effects on earthquake rupture. However, studies for understanding these effects have only recently begun (Harris and Day, 1997).

To date, earthquake rupture processes have usually been considered to be on the fault, which is a smooth, planar surface embedded in a uniform elastic medium. Slip-weakening

Copy right(C) The Society of Geomagnetism and Earth, Planetary and Space Sciences (SGEPSS); The Seismological Society of Japan; The Volcanological Society of Japan; The Geodetic Society of Japan; The Japanese Society for Planetary Sciences. and/or rate and state dependent friction laws have been introduced on such planar surfaces to evaluate fracture energies of earthquake faulting (Ida, 1972; Aki, 1979), the acceleration of fault rupture propagation (Andrew, 1976a, b; Ohnaka, 1993; Shibazaki and Matsu'ura, 1998), and relatively long term earthquake cycles (Stuart et al., 1985; Tse and Rice, 1986). In these studies, a characteristic slip distance $d_{0}$, or a cohesive zone size $w_{0}$, is a key parameter for the above evaluations of the slip-weakening and/or rate and state dependent friction laws.

The basic idea of these friction laws follows Barenblatt (1959) who introduced the concept of a cohesive zone around the tip of a crack so that all stresses are finite at the crack tip. In this concept, the crack tip is never perfectly sharp and inelastic behavior at high stress levels near the crack tip is the cause of a plastic deformation zone with finite stresses. It is noted that the plastic deformation zone is distributed not only in the crack plane but in the volume around the crack tip. Thus, the cohesive zone size, $w_{0}$, should be related to the width of the LVZ depicted by fault zone trapped waves.

In this paper we first review the analyses of fault-zone trapped waves to evaluate the widths of the LVZ of natural earthquake faults. A model of earthquake rupture with the LVZ related to the plastic deformation zone is introduced from a fracture mechanics view point. We analyze the model to obtain quantitative relationships between the width of the LVZ and the fracture mechanics parameters such as a breakdown stress drop, the characteristic slip distance and static stress drop. 
Table 1. Results for the trapped wave analysis for various faults.

\begin{tabular}{|c|c|c|c|c|}
\hline Active fault or earthquake fault & Magnitude & width of LVZ & reduction of $V s$ & $Q$ \\
\hline the 1992 Landers earthquake ${ }^{1}$ & 7.4 & $100-250 \mathrm{~m}$ & $35-45 \%$ & $20-60$ \\
\hline the 1995 Hyogo-ken Nanbu earthquake ${ }^{2}$ & 7.2 & $50 \mathrm{~m}$ & $50 \%$ & 10 \\
\hline the 2000 Tottori-ken Seibu earthquake ${ }^{3}$ & 7.3 & $>50 \mathrm{~m}$ & $30 \%$ & - \\
\hline San Jacinto fault ${ }^{4}$ & - & $120 \mathrm{~m}$ & $25 \%$ & - \\
\hline Mozumi-Sukenobu fault ${ }^{5}$ & - & $30-200 \mathrm{~m}$ & $40 \%$ & - \\
\hline
\end{tabular}

${ }^{1}$ Li et al. (1994) ${ }^{2}$ Ito and Kuwahara (1996) ${ }^{3}$ Kuwahara et al. (2001) ${ }^{4}$ Li et al. (1997) ${ }^{5}$ Ito et al. (2002).

\section{LVZ's from Trapped Waves}

Fault-zone trapped waves have, so far, been reported for the aftershock regions immediately after large earthquakes, such as the 1992 Landers earthquake (Li et al., 1994), the 1995 Hyogo-ken Nanbu earthquake (Ito and Kuwahara, 1996), and the 2000 Tottori-ken Seibu earthquake (Kuwahara et al., 2001). The trapped waves are also observed not just after the large earthquake, but for more than 100 years after the large earthquake. Li et al. (1997) reported that the fault zone trapped waves are observed in a region of a seismic gap of the San Jacinto fault. Ito et al. (2002) show that the trapped wave is found at the Mozumi-Sukenobu fault which has not been activated for at least 140 years (Takeuchi et al., 2002). This evidence suggests that the LVZ exists from the surface to the seismogenic depth for about 100 years with large S-wave velocity reductions compared with the surrounding rock. The properties such as a width, velocity reduction and Q of the LVZ obtained by the trapped wave analysis are compiled in Table 1.

Fault-zone trapped waves have, thus, been observed above several major faults. The widths range from a few tens of meters to a few hundred meters. It is also suggested that the LVZ's are stable for a period of at least 100 years.

\section{A Model of Rupture Process within a Fault LVZ}

A plastic deformation occurs at the vicinity of a crack tip because stress concentrated at the crack tip exceeds the yielding stress of rock material. Supposing that the fault rupture propagation can be treated as the shear crack propagation, a fault damaged zone is, thus, related to the plastic deformation at the vicinity of the tip of propagating fault fracture. The width of the damaged zone, i.e. fault LVZ, can be regarded as approximately the size of the plastic deformation zone at the crack tip, considering that the damaged zone of the Nojima fault shows the plastic deformation (Tanaka et al., 2001).

Based on this model, the earthquake fault is treated as a propagating Mode III (anti-plane shear) crack because the Mode III crack model is simple to analyze and sufficient to understand the basic process of the crack propagation. The singular stress field at the crack tip in the Cartesian coordinate system in Fig. 1 can be written as

$$
\begin{aligned}
\tau_{x z} & =K_{\mathrm{III}} /(2 \pi r)^{1 / 2}(-\sin (\theta / 2)) \\
\tau_{y z} & =K_{\mathrm{III}} /(2 \pi r)^{1 / 2}(\cos (\theta / 2))
\end{aligned}
$$

where $K_{\mathrm{III}}, r$ and $\theta$ are stress intensity factor for Mode III crack, distance from the crack tip and an angle from the

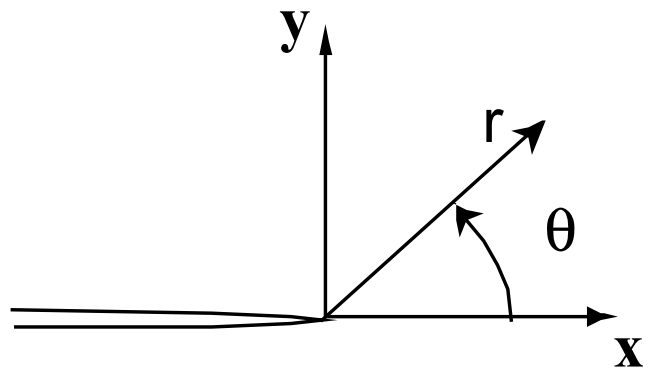

Fig. 1. The coordinate system at the crack tip.

$x$-axis, respectively. For a crack with edges at $x= \pm a$ and with a static stress drop $\Delta \tau, K_{\mathrm{III}}=\Delta \tau(\pi a)^{1 / 2}$. The complete stress fields in the vicinity of the crack tip consists of this singular field plus a uniform stress field $\tau_{f}$, where $\tau_{f}$ is a sliding friction level (Rice, 1980). Given that the uniform stress field of the sliding friction level does not affect the following discussion, only the singular stress field will hereafter be considered.

The area of the plastic deformation is assumed to be given by the yield criterion of von Mises (e.g. Schmidt and Rossmanith, 1983). The yield criterion for Mode III is simply given by

$$
\tau_{x z}^{2}+\tau_{y z}^{2}=\tau_{y s}^{2}
$$

where $\tau_{y s}$ is the yield stress in the case of simple shear. It is noted that $\tau_{y s}$ in this expression is the breakdown stress drop defined in the slip-weakening friction law. From the equations (1) and (2), the shape of the plastic deformation area is a circle with a radius $W_{p}$, where

$$
W_{p}=\left(K_{\mathrm{III}} / \tau_{y s}\right)^{2} / 2 \pi \text {. }
$$

The relation between the stress field and plastic deformation is illustrated in Fig. 2. The fault damaged zone produced by the past earthquakes should act as the plastic deformation zone. The fault damaged zone is indicated by cross hatched area.

Based on this model, we can propose methods to obtain the values of the slip-weakening friction parameters on natural faults from the observed width of the fault low velocity zone. For example, we adopt the model parameters as $W_{p}=50 \mathrm{~m}$ for the Nojima fault from the trapped wave observation (Ito and Kuwahara, 1996) and $a=10 \mathrm{~km}$ as a representative value of the fault length measured from 


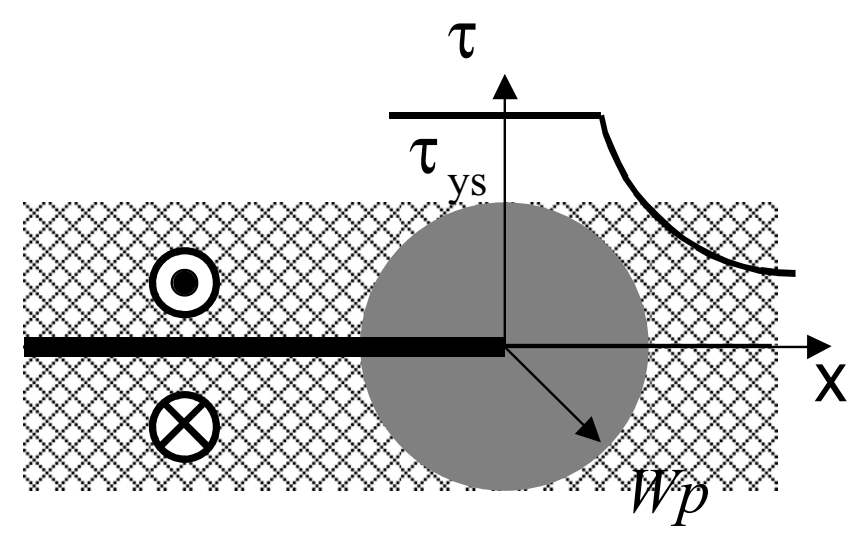

Fig. 2. A model of breakdown process around the crack tip within the fault damaged zone. The zone of the plastic deformation is shown by the dark shaded area. The shape of the zone is a circle for a Mode III crack. The fault damaged zone is indicated by a cross hatched zone which is also the plastic deformation zone.

the hypocenter depth of $14.3 \mathrm{~km}$ (determined by the Japan Meteorological Agency). In this case, equation (3) gives $\tau_{y s}=10 \Delta \tau$. This means that the breakdown stress drop defined in the slip-weakening friction law is 10 times larger than the static stress drop. Rice (1980) shows that the characteristic slip distance $d_{0}$ in the friction law is related to $W_{p}$ as

$$
W_{p}=9 \pi d_{0} \mu / 16 / \tau_{y s}
$$

where $\mu$ and $\tau_{f}$ are the shear modulus of a material and the sliding friction, respectively. For the Nojima fault, $d_{0}$ is estimated from Eq. (4) to be about $10 \mathrm{~cm}$, assuming $\Delta \tau=5$ $\mathrm{MPa}$ from an average value determined from the seismic observation by Ide and Takeo (1997). Thus, the breakdown stress drop and the characteristic slip distance can be estimated from the width of the fault LVZ. These estimates will be compared in the following discussion with estimates from the seismic waveform data.

Ide and Takeo (1997) estimated $d_{0}$ to be in the range from $0.5 \mathrm{~m}$ to $1 \mathrm{~m}$ for the Nojima fault. However, they mentioned that these values are only an upper limit because the results were obtained from a limited frequency band in the waveform data and inplementing smoothing constraint. Guatteri et al. (2001) inferred a lower bound of $d_{0}$ of about $16 \mathrm{~mm}$ from rather stable estimate of fracture energy, $G=$ $\tau_{y s} d_{0} / 2$, and the upper bound of the rock strength. Guatteri et al. (2001) suppose the upper bound of the rock strength to be a static coefficient of friction of 0.7 , applicable to the intact host rock. This means that the upper bound of $\tau_{y s}$ is 175 $\mathrm{MPa}$ at $10 \mathrm{~km}$ depth. Thus, the estimates from the present model are consistent with the methods using the seismic waveforms. This indicates that the present model can give a reasonable value of the slip-weakening parameters. It should be noted that the width of the fault low velocity zone is a parameter observable from the trapped wave even before a large earthquake, as indicated in the previous section.

\section{Concluding Remarks}

Analyses of the fault-zone trapped waves have shown that widths for fault LVZ's range from values of the order of
$10 \mathrm{~m}$ to a few hundred meters. A model where the fault LVZ acts as the plastic deformation zone around the crack tip is proposed to evaluate the earthquake rupture processes within the fault LVZ. The earthquake rupture process is regarded as Mode III crack propagation in the model. The model provides a simple relationship between the width of the LVZ, the breakdown stress drop at the crack tip and the characteristic slip distance. The parameters applicable to the Nojima fault show that the breakdown stress drop is 10 times larger than the static stress drop and the characteristic slip distance, $d_{0}$, is about $10 \mathrm{~cm}$, consistent with previous results estimated from the seismic waveform data. Thus, the trapped wave observation has great potential for predicting a process for large earthquakes.

Acknowledgments. We wish to thank our reviewers, T. Stern and K. Ito, for the helpful comments. We also thank A. Stork for the improvement of the manuscript.

\section{References}

Aki, K., Characterization of barriers on an earthquake fault, J. Geophys. Res., 84, 6140-6148, 1979

Andrew, J., Rupture propagation with finite stress in antiplane strain, $J$. Geophys. Res., 81, 3575-3582, 1976a.

Andrew, J., Rupture velocity of plane strain shear cracks, J. Geophys. Res., 81, 5679-5687, 1976b.

Barenblatt, G. I., The formation of equilibrium cracks during brittle fracture General idea and hypothesis, J. Appl. Math. Mech., 23, 622-636, 1959.

Fujita, K. and O. Ikuta, Resistivity structure of the central part of the Yamasaki fault studied by the multiple electrodes resistivity method, Earth Planets Space, 52, 567-571, 2000.

Guatteri, M., P. Spudich, and G. C. Beroza, Inferring rate and state friction parameters from a rupture model of the 1995 Hyogo-ken Nanbu (Kobe) Japan earthquake, J. Geophys. Res., 106, 26511-26521, 2001.

Harris, R. and S. Day, Effect of a low-velocity zone on a dynamic rupture, Bull. Seism. Soc. Am., 87, 1267-1280, 1997.

Ida, Y., Cohesive force across the tip of a longitudinal-shear crack and Griffith's specific surface energy, J. Geophys. Res., 77, 3796-3805, 1972.

Ide, S. and M. Takeo, Determination of constitutive relation of fault slip based on seismic wave analysis, J. Geophys. Res., 102, 27379-27391, 1997.

Ito, H. and Y. Kuwahara, Trapped waves along the Nojima fault from the aftershock of Kobe earthquake, 1995, Proceedings of VIIIth International Symposium on the observation of the Continental Crust Trough Drilling, 399-402, 1996.

Ito, H., Y. Kuwahara, T. Miyazai, O. Nishizawa, T. Kiguchi, K. Fujimoto, Y. Ohtani, H. Tanaka, T. Higuchi, S. Agar, A. Brie, and H. Yamamoto, Structure and physical properties of the Nojima fault by the Active fault drilling, Geophys. Explor, 49, 522-535, 1996.

Ito, H., K. Nishigami, and Y. Kuwahara, Fault guided waves at the MozumiSukenobu fault, central Japan, Geophys. Res. Lett., 2002 (in preparation)

Kuwahara, Y., K. Imanishi, and H. Ito, Seismic trapped wave observation in the fault zone of the Western Tottori earthquake of 2000, Program abstract of Japan Earth and Planetary Sci. Joint meeting, 2001.

Li, Y.-G., P. C. Leary, K. Aki, and P. E. Malin, Seismic trapped modes in the Oroville and San Andreas fault zones, Science, 249, 763-766, 1990.

Li, Y.-G., K. Aki, D. Adams, and A. Hasemi, Seismic guided waves trapped in the fault zone of the Landers, California, earthquake of 1992, J. Geophys. Res., 99, 11705-11722, 1994.

Li, Y.-G., K. Aki, and F. L. Vernon, San Jacinto fault zone guided waves: A discrimination for recently active fault strands near Anza, California, $J$. Geophys. Res., 102, 11689-11701, 1997.

Mooney, W. D. and A. Ginzburg, Seismic measurements of the internal properties of fault zones, Pure Appl. Geophys., 124, 141-157, 1986.

Ohnaka, M., Critical size of the nucleation zone of earthquake rupture inferred from immediate foreshock activity, J. Phys. Earth, 41, 45-46, 1993.

Rice, J. R., The mechanics of earthquake rupture, in Physics of the Earth's Interior, edited by A. Dziewonski and E. Boshi, pp. 555-649, Italian 
Physical Society, Amsterdam, North Holland, 1980.

Schmidt, R. A. and H. P. Rossmanith, Basics of rock fracture mechanics, in Rock Fracture Mechanics, edited by H. P. Rossmanith, pp. 1-29, Springer, Verlag, 1983.

Shibazaki, B. and M. Matsu'ura, Transition process from nucleation to highspeed rupture propagation: scaling from stick-slip experiments to natura earthquakes, Geophys. J. Int., 132, 14-30, 1998.

Stuart, W. D., R. J. Archuleta, and A. G. Lindh, Forecast model for moderate-earthquake near Parkfield, California, J. Geophys. Res., 90, 592-604, 1985.

Takeuchi, A., H. Ongirad, and T. Akimitsu, Recurrence interval of big earthquakes along the Atotsugawa fault system, central Japan-Results of Seismo-geological survey, Geophys. Res. Lett., 2002 (submitted).
Tanaka, H., K. Fujimoto, T. Ohtani, and H. Ito, Structural and chemical characterization of shear zones in the freshly activated Nojima fault, Awaji island, southwest Japan, J. Geophys. Res., 106, 8789-8810, 2001. Tse, S. T. and J. R. Rice, Crustal earthquake instability in relation to the depth variation of frictional slip properties, J. Geophys. Res., 91, 9425$9472,1986$.

Unsworth, M., G. Egbert, and J. Booker, High-resolution electromagnetic imaging of the San Andreas fault in Central California, J. Geophys. Res. 104, 1131-1150, 1999

Y. Kuwahara (e-mail: y-kuwahara@aist.go.jp) and H. Ito 